\title{
Analysis on Network-based Sensor Technology
}

\author{
Wang Fei \\ Nanchang Institute of Science and Technology, Nanchang China, 330108
}

Keywords: network sensor, wireless sensor, wired sensor, technical analysis

\begin{abstract}
Now, with the rapid development of science and technology in China, computer networking technology is leading the development of the time, giving a strong support for people's daily life. Various traditional industries have been integrated with computer technology, which is an evitable trend in line with the times. And sensor is an indispensable device for information collection, which is widely applied in other industries. The paper, thus, analyzed the network-based sensor technology.
\end{abstract}

At present, computer technology as well as networking technology have exerted huge impact on all walks of life, and in the process, and network-based testing technology has appeared with the combination of automatics and network technology, which can achieve remote testing with an ideal effect in the system testing. In the test, information gathering is an evitable link of the sensor, therefore, the term of network-based technology rises so as to release and share information in the areas covered by the network. [1]

\section{The basic introduction to network-based sensor}

The design goal of network-based sensor is to integrate advanced computer information technology into the application of sensors. The sensor is linked by modularized structure, the simulation signals output by sensitive elements after the phase flow are processed by related data system. Then, they are bound to be frames by network protocol, which are transmitted to network by the network interface after adding accurate numbers and address. Meanwhile, network processing units are able to accept data and information transmitted by other interfaces in the network. In this way, sensor has become an independent site in the measurement net as shown in figure 1.

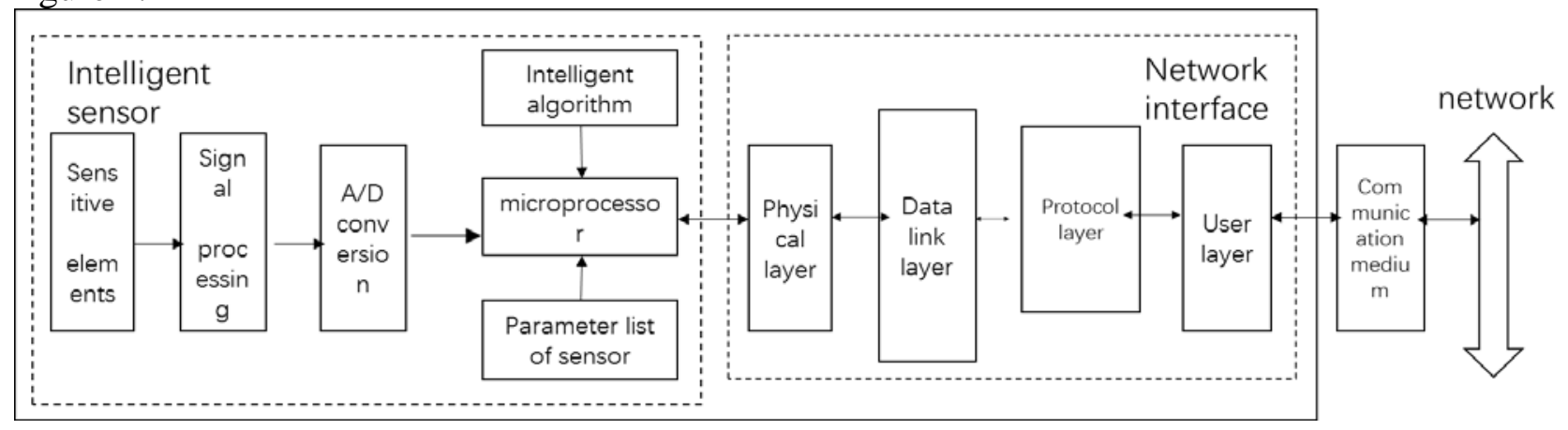

Figure 1 Basic structure of network-based sensor

As early as 1994, scholars in the industry published academic papers on structure standard of smart sensors in which they classified all sensors accurately, ensuring their practicability as well as proposing technological innovation, storing all data uniformly. In this way, most data information can be accessed via the network protocol. And network processors and data model converter are utilized in most cases, going beyond specific networks, which marks a technological upgrading. The standardized interface processor with innovation can make average sensors access the network, and make regional network an independent unit. In addition, network node is featured by configuration as well as interoperability. [2] In current stage, network-based sensors can be divided into two types: 
wired network-based sensor and the wireless network-based sensor.

\section{Wired network-based sensor}

Wired network-based sensor based on IEEE 1451.2 standard merely makes a definition of interface logic and the form of TEDS, while other parts are automatically achieved by sensor manufacturers in order to ensure the competitiveness of the equipment in performance and quality. At current stage, wired network sensors have been applied more widely, which means an advance of computer network technology in China. At the same time, the standard also establishes a standard interface, connecting the interface model of intelligent transmitter, STIM, an independent interface of the transmitter, connecting two nodes, achieving notable effect in practical application. (See figure 2) [3]

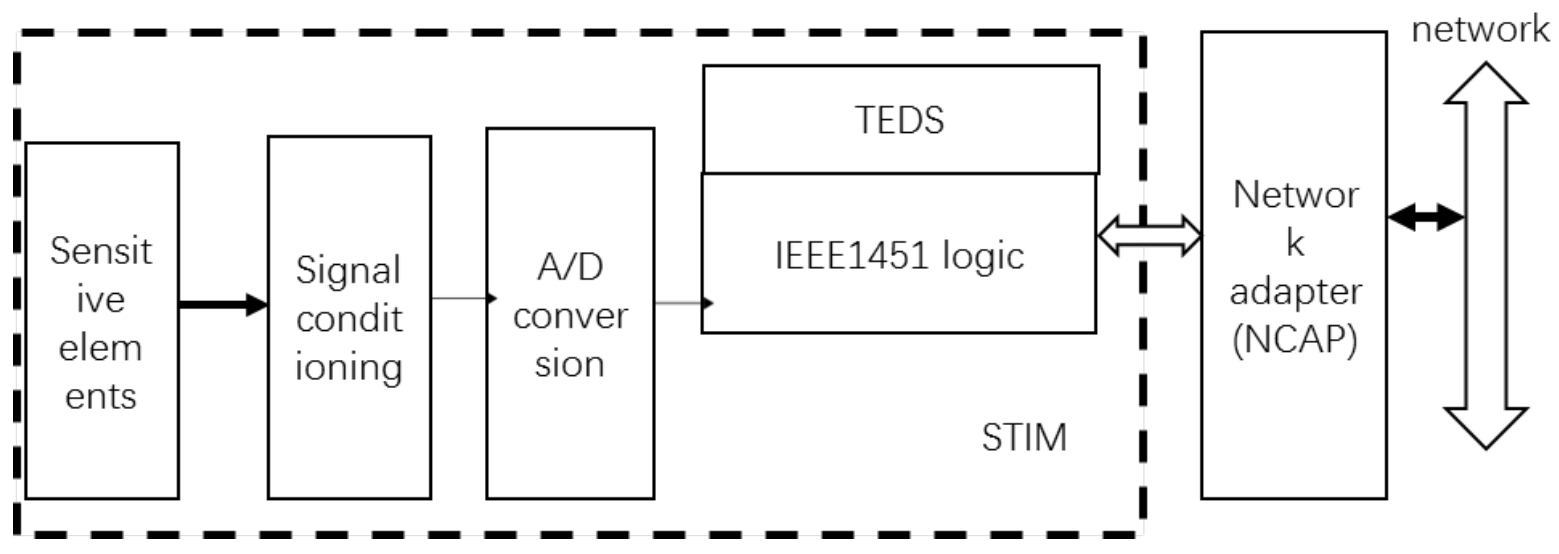

Figure 2 Structure of network sensor based on IEEE 1451

Transmitter model should have internal information up to standard, such as manufacturers, data code, serial number, limit of user. In the actual operation, when the power supply is added STIM, the understanding and use of data should be paid attention, which will provide comprehensive technical base for the NCAP technology, coordinate each other in the work.

When the NCAP accesses the next TEDS data, the communication speed and the environment of data will change. So NCAP can know the communication speed as well as the data format of transmitter in the passage. In general, 12-bit or 16-bit format is adopted. Moreover, physical unit of the measured object can be obtained accurately. According to relevant operational rule, it can be converted into international unit, offering help for the future work.

Generally, there are 8 units which can be found in the electronic data sheet of transmitter, among which, two are perquisites for the equipment operation, while others can be chosen in light of real condition of the transmitter for the expansion of information data. In the process, it is necessary to conduct a comprehensive technical work of TEDS technology in order to regulate the data structure and the time limit. In regulating, objects involved should be classified rationally, including the uncertainty of the scope of the object, bound, as well as the research on data model. In the calibration of TEDS, each information channel includes an object, and checks parameters like date, calibrating circle, which supports multiple-node model. And the function expansion is completed by the 1451.2 standard for the applications in industry.

As for the STIM channel, mathematical model of each channel is expressed by polynomial function. In order to efficiently avoid the too large number of polynomials, its curve will be divided into several segments, and it is important to demonstrate the number of variables in each segment as well as the drift value and the number of coefficients. Then, standard calibration methods will identify the corresponding strategies. [6] In the current stage, standard network sensors are widely applied in China, which realize ideal effect in the practice, particularly the application of STIM and the NCAP interface has achieved a high technical standard. The hardware can use dedicated chips to operate, while software module includes SIM module, sensor interface module and TII module. 


\section{Bluetooth wireless network sensor}

Now, with the wide application of wireless sensors, Bluetooth wireless sensors have brought great convenience for people. In most measurement and control environments, people focus on the readiness of the measurement and control, and hence, the Bluetooth wireless sensor is born at the right time, which is closely associated with people's subjective needs. As for traditional wired sensors, there is inconvenience in the real operation, especially in remote regions or areas sparsely populated. As long as there are no permanent personnel to operate, the ideal effect is hard to be realized. [7] In such period, some scientists proposed large-scale application of wireless sensors when the technology was not mature, and people has poor understanding about it. When the real research work station opens, some problems will appear, posing a huge challenge for researchers of China. While the invention of wireless network sensors has efficiently addressed the problems in the operation of the wired network sensors, breaking through the technology limitations in a large degree, marking the turning point of the network sensors in China. When wireless network sensor is formally put into place, it transforms people's life, study and work, which is a major achievements of the wireless sensor technologies. [8]

Bluetooth technology, a low-power short-range wireless information transmission device developed by several American scientific and technological corporations in May 1998, was popular with the market at that time. The principle of the technology is to ensure the efficient transmission of information by the openness of speech information as well as the data wireless transmission. Such transmission is efficient only in short range, but in the interpretation, it will be influenced by external environment. When walls or other objects hinder the transmission channel, for instance, the integrity of information transmission will be greatly reduced, causing the information delay. What is more, the strengths of Bluetooth technology outweigh its defects, and the working frequency range of Bluetooth devices is universal worldwide and portable with convenient use, security encryption, and anti-interference capabilities, which can finish multiple links. [9]

In data transmission, wireless sensor is different from traditional wired sensors, with the former taking data transmission as the center, while the latter as the aim. Now, wireless network sensors mainly transform basic transmission ideas, opening a new horizon for the technological development. The essence is to regard the sensor as an important equipment for sensing data flow or data source, and the network as the data space or the sensing library. Besides, the transmission safety of wireless network sensor is another important issue. In the transmission, wireless transmission equipment will be influenced by external environment or the transmission media, which brings different impacts on the transmission. Now, all network safety mechanisms are not suitable for application in the field, therefore, new network safety protocol will be formulated for wireless sensors in the development. As for large-scale use of network equipment, safety of information transmission should be taken into account. Thus, it is necessary to start from the preservation of router safety to find possible safe routes to ensure the security of network running. Then, the focus should be paid on safety protocol construction, safety encryption measures for data, minimizing the damages or loss in data transmission.

Currently, wireless network sensor based on Bluetooth protocol mainly consists of STIM, Bluetooth module and NCAP as shown in figure 3 .

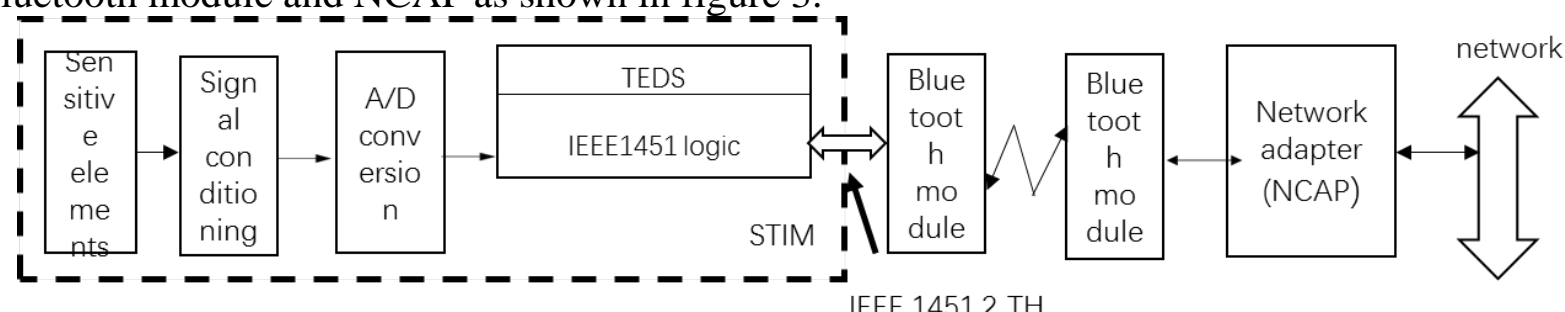

Figure 3 Structure of wireless network sensors

In the interface of 10 line TII among Bluetooth modules, they are connected with equipment by TII interface so as to solve problems in the transmission, assign and accept tasks for remote control. 
Compared with standard wired network sensors, wireless network sensors only increase two Bluetooth modules, so there is no need to discuss it in detail. The Bluetooth nodule is the major innovation trend in current network sensors, therefore, a Bluetooth circuit similar to TII interface should be established, in this way, a specialized processor can be set up to process information system. In foreign countries, many scientific and technological companies have utilized the proposed method to produce equipment, bringing much convenience for the actual work. Meanwhile, due to this technological innovation, it has promoted the development of wireless network sensors in China.

\section{The structure of network sensor in its network system}

The main goal of measuring servers is to accurately distribute tasks for all basic functions to make them purposeful in actual work, which realizes the computing of collected data in units to some extent, and comprehensively processes the computing results, achieves short-term storage and printing of data.

The main function of measuring browsers is to provide interface for Web browsers or other software, measure nodes on the scene, analyze and process measuring results. [10]

In the overall running of system, sensors can not only conduct information exchange with measuring servers, but complete information conversion between sensors conforming to IEEE1451 standard and the actuator, which marks a new breakthrough of the industry. The regular information exchange can change the monotony of the transmission system in a large degree, and cut down the information content in the network system, which has been widely applied, and realized good effect. It is vital to ensure the timeliness of the running system, which improves the efficiency of the operating system and finishes more tasks in the same time.

\section{Conclusions}

The release of IEEE1451.2 standard has largely promoted the development and application of standard network sensors, bringing new opportunities for the development of sensors. Moreover, with the increasing advance of

Bluetooth technology in China, the application of network sensors has reached a new high. The advent of wireless network sensors has replaced traditional ones, which are more convenient, faster in the form, bringing better life for people, and enormously enhancing the working efficiency. Over the past few years, its advantages over technology have gradually demonstrated. The paper, analyzed the network sensor technology, put forward its unique views considering the practical development as well as the work experience, hoping to provide some help for the future work.

\section{References}

[1] Li Xunwen. Sensor development based on network virtual instrument technology [J]. Experimental Technology and Management, 2013,15(2):81-84.

[2] Li Fengbao, Liu Jin, Gu Tianxiang. Research on network sensor technology [J]. Sensor Technology, 2015,36(7):62-64.

[3] Han Hongyan. Research on wireless network sensor technology [J]. Computer Knowledge and Technology (academic exchanges), 2013,20(1):117-118.

[4] Xu Dingcheng, Wang Qihui, Lu Weiguo. Network sensor and its application based on Bluetooth technology [J]. MEASUREMENT \& CONTROL TECHNOLOGY, 2014,30(1):37-39,45.

[5] Zhang Ming, Li Zhengxiao, Xiao Jianjun. The latest development of network sensor technology based on IEEE 1451 standard. [J] Instrument Standardization \& Metrology, 2017,21(5):20-23,26.

[6] Xie Chenghan, Lu Saijie, Wang Hao, et al. Event-driven output feedback control in wireless sensor networks. Journal of Computer Research and Development, 2017,12(11):2639-2645. 
[7] Li Xiaoyu, Gao Yi, Li Ming, et al. The network interface design of time grid displacement sensor[J] MEASUREMENT \& CONTROL TECHNOLOGY, 2017,10(7):110-115.

[8] Xiang Xinjian. Research on sensor network technology based on RS-485 bus. [J]. Sensor Technology, 2014,19(8):14-16.

[9] Wang Yangyang, Peng Donglin, Chen Xihou, et al. Research on grid displacement sensor based on "Internet + " thinking [J] Journal of Chongqing University of Technology (nature science), 2016,18(4):116-119,131.

[10] Li Fengbao, Liu Jin. Network sensor technology [C]. Annual Report of China Academy of Engineering Physics (2014) 2014. 